\title{
Characterising the performance of balanced memory networks
}

\author{
Alex Metaxas", Reinoud Maex, Rod Adams, Volker Steuber, Neil Davey \\ From Twenty Second Annual Computational Neuroscience Meeting: CNS*2013 \\ Paris, France. 13-18 July 2013
}

In previous work [1], we investigated the associative memory performance of networks of Izhikevich neurons with various synaptic plasticity regimes. However, the firing rates observed were far higher than those observed in vivo. In their recent Science paper [2], Vogels et al. describe a model of associative memory that exhibits biologically plausible firing rates, using a network of Integrate and Fire (IAF) neurons in which the inhibitory to excitatory synapses are plastic. Their self-organising learning rule provides a homeostatic function, leading to balanced excitation and inhibition. Further, by achieving a globally balanced state, the network displays asynchronous irregular dynamics. This sparse pattern of activity, which is present in cortical networks in vivo, enables rapid responses to small changes in the input [2].

The patterns are stored via a simplified form of one-shot Hebbian learning of synapses between excitatory neurons. The plastic inhibitory to excitatory synapses serve to balance the excitation in the memory assembly, mirroring the potentiated excitatory synapses and thereby allowing the stored pattern to be suppressed when not activated by external stimuli. This is a feature lacking in the ANN attractor networks we have studied previously [1], where the activation of a pattern causes the network to reach a fixed point.

In this work, we characterise the performance of the Vogels' network using a metric, Effective Capacity, adapted from [3]. Vogels uses a random architecture, with each neuron having a 0.02 probability of being connected to any other neuron. However, analyses of the connectivity of cortical networks have suggested that they may have nonrandom features [4]. Having measured the performance of the network with random connectivity, we measure the effect on performance of a small world architecture [3].

\footnotetext{
* Correspondence: a.metaxas@herts.ac.uk

Science and Technology Research Institute, University of Hertfordshire, Hatfield, Hertfordshire, AL10 9AB, UK
}

(c) 2013 Metaxas et al; licensee BioMed Central Ltd. This is an Open Access article distributed under the terms of the Creative Commons Attribution License (http://creativecommons.org/licenses/by/2.0), which permits unrestricted use, distribution, and reproduction in any medium, provided the original work is properly cited.
These results are contrasted with a similar study of connectivity patterns in (non-spiking) ANN networks [5].

Published: 8 July 2013

\section{References}

1. Metaxas A, Maex R, Adams R, Steuber V, Davey N: Determinants of associative memory performance in spiking and non-spiking neural networks with different synaptic plasticity regimes. BMC Neuroscience 2012, 13(Suppl 1):P156.

2. Vogels TP, Sprekeler H, Zenke F, Clopath C, Gerstner W: Inhibitory Plasticity Balances Excitation and Inhibition in Sensory Pathways and Memory Networks. Science 2011, 334:1569-1573.

3. Davey N, Calcraft L, Adams R: High capacity, small world associative memory models. Connection Science 2006, 18:247-264.

4. Song S, Sjöström PJ, Reigl M, Nelson S, Chklovskii DB: Highly Nonrandom Features of Synaptic Connectivity in Local Cortical Circuits. PLoS Biol 2005, 3:e68.

5. Chen W, Maex R, Steuber V, Davey N: Clustering predicts memory performance in networks of spiking and non-spiking neurons. Front Comput Neurosci 2011, 5:14.

doi:10.1186/1471-2202-14-S1-P88

Cite this article as: Metaxas et al:: Characterising the performance of balanced memory networks. BMC Neuroscience 2013 14(Suppl 1):P88. and take full advantage of:

- Convenient online submission

- Thorough peer review

- No space constraints or color figure charges

- Immediate publication on acceptance

- Inclusion in PubMed, CAS, Scopus and Google Scholar

- Research which is freely available for redistribution 